\title{
Poliartrite e Nódulos Subcutâneos como Primeira Manifestação de Carcinoma de Pâncreas
}

\section{Polyarthritis and Subcutaneous Nodules as First Manifestation of Carcinoma of Pancreas}

\author{
Raquel Godinho de Sá(1), Calina Maria Loures de Oliveira Teixeira ${ }^{(1)}$, Luana Gerheim Machado ${ }^{(2)}$, Herval de \\ Lacerda Bonfante $^{(3)}$, Ana Maria Coimbra Chaves ${ }^{(4)}$, Júlio Maria Fonseca Chebli( ${ }^{(5)}$, Ricardo Baesso de Oliveira ${ }^{(6)}$
}

\section{RESUMO}

A associação de poliartrite, nódulos subcutâneos e carcinoma de pâncreas é uma condição rara e pouco diagnosticada. O presente trabalho tem o objetivo de relatar um caso de carcinoma pancreático associado a poliartrite e nódulos subcutâneos, semelhantes ao eritema nodoso, cujo exame histopatológico evidenciou tratar-se de paniculite com necrose gordurosa. Os autores pretendem, por meio deste relato, lembrar essa associação incomum que se torna de grande importância clínica, considerando que artrite e nódulos subcutâneos podem anteceder as manifestações clínicas de neoplasia pancreática.

Palavras-chave: artrite, nódulos subcutâneos, carcinoma de pâncreas.

\section{INTRODUÇÃO}

A associação de necrose gordurosa e doença pancreática foi primeiramente descrita por Hansemann, em 1882, e Chiari, em 1883. Em 1908, Berner relatou síndrome similar, porém associada com malignidade pancreática ${ }^{(1-5)}$. Em 1914, Jenckel relatou o componente articular como parte dessa síndrome. Artrite, paniculite e doença pancreática têm sido encontradas formando uma rara tríade, ocorrendo em menos de $1 \%$ dos pacientes com pancreatite aguda ou carcinoma pancreático ${ }^{(1,6)}$.

Poliartrite pode ocorrer como manifestação de vários processos patológicos, podendo ser encontrada em associação à doença pancreática ${ }^{(1)}$, porém raramente o quadro de artrite antecede o diagnóstico de uma doença pancreática silenciosa ${ }^{(2,3)}$.

\begin{abstract}
The association of polyarthritis, subcutaneous nodules and carcinoma of the pancreas is a rare and underdiagnosed condition. The present work aims to report a case of a patient with carcinoma of pancreas associated with polyarthritis and subcutaneous nodules, similar to erythema nodosum, with histopathological findings of panniculitis with fat necrosis. The authors emphasize the importance of this uncommon association, considering that polyarthritis and subcutaneous nodules may appear before the clinical manifestations of the carcinoma of the pancreas.
\end{abstract}

Keywords: arthritis, subcutaneous nodules, carcinoma of the pancreas.

Necrose gordurosa subcutânea tem sua origem ainda pouco esclarecida. Sugere-se que possa estar relacionada a um processo de degradação do tecido adiposo pela liberação de enzimas pancreáticas. Sua forma mais comum de apresentação clínica é como nódulos eritematosos, ocorrendo em $2 \%$ a 3\% de todos os pacientes com doença pancreática ${ }^{(7)}$.

Os autores relatam um caso de neoplasia pancreática, em que artrite e lesões eritematosas nodulares precederam o diagnóstico de doença pancreática.

\section{RELATO DE CASO}

Paciente do sexo masculino, branco, 63 anos, aposentado, natural de Recife, residente em Muriaé (MG), diabético, tabagista e com história de etilismo, foi internado com quadro de artrite em articulações de punhos, joelhos,

Recebido em 23/03/07. Aprovado, após revisão, em 06/06/07. Declaramos a inexistência de conflitos de interesse.

Trabalho realizado no Serviço de Reumatologia da Santa Casa de Misericórdia de Juiz de Fora (SCMJF).

1. Graduanda de Medicina da Universidade Fundação Oswaldo Aranha (UNIFOA) e interna da SCMIF.

2. Graduanda de Medicina da Universidade Federal de Juiz de Fora (UFJF) e estagiária do Serviço de Reumatologia da SCMJF.

3. Professor-assistente do Departamento de Farmacologia da UFJF, reumatologista e chefe do Serviço de Reumatologia da SCMJF.

4. Médica dermatologista da SCMIF.

5. Professor adjunto do Departamento de Clínica Médica da Disciplina de Gastroenterologia da UFJF.

6. Reumatologista e coordenador do Internato de Medicina da SCMIF.

Endereço para correspondência: Herval de Lacerda Bonfante, Rua Capitão Arnaldo de Carvalho, 693/301, CEP 36036-180, Juiz de Fora, MG, Brasil, e-mail: hervalbonfante@ terra.com.br 
primeira metatarsofalangiana e tarso bilateralmente. Sentia dor de grande intensidade, eritema e calor havia aproximadamente 3 semanas. Relatava febre em torno de $38^{\circ} \mathrm{C}$, com freqüência diária, e lesões nodulares, eritematosas e dolorosas ( 2 a $3 \mathrm{~cm}$ de diâmetro) localizadas principalmente em membros inferiores, mas que também acometiam membros superiores, semelhantes ao eritema nodoso.

Não havia queixa em relação aos sistemas respiratório, digestivo nem urinário. Os exames complementares revelavam discreta anemia (hemoglobina $=11,1 \mathrm{~g} / \mathrm{dl}$ ) e leucocitose (leucometria global $=25.200$ céls.$/ \mathrm{mm}^{3}$ ), sem desvio à esquerda, e elevação significativa da velocidade de sedimentação das hemácias (108 mm na primeira hora) e proteína C reativa $164 \mathrm{mg} / \mathrm{l}$ (normal até $6 \mathrm{mg} / \mathrm{l}$ ).

Aventou-se a hipótese de artrite infecciosa ou quadro articular reacional. Artrocentese não obteve líquido sinovial para culturas e demais exames de rotina. Iniciou-se tratamento com ceftriaxona, oxacilina e analgésicos. Após 10 dias de tratamento parenteral, não havia nenhum sinal de melhora, continuando com poliartrite dolorosa e os nódulos subcutâneos eritematosos dolorosos. Optou-se por iniciar terapia com glicocorticóide (prednisona $40 \mathrm{mg} /$ dia), que também não alterou a evolução do quadro.

Biópsia de nódulo de membro inferior revelou paniculite mista do tipo necrose gordurosa.

Baseando-se nesse resultado, sugeriu-se pesquisa de doença pancreática como causa de nódulo subcutâneo e presença de necrose gordurosa. Não havia sintomas abdominais e a ultra-sonografia abdominal total mostrou-se normal. A dosagem das enzimas pancreáticas mostrou os seguintes resultados: amilase de $2.847 \mathrm{U} / 1$ (valor normal: 25 a $125 \mathrm{U} / \mathrm{l}$ ) e lipase de $280 \mathrm{U} / \mathrm{l}$ (valor normal: 2 a 18 $\mathrm{U} / \mathrm{l}$ ). Prosseguindo a investigação, realizou-se tomografia computadorizada de abdome, que evidenciou calcificações e a presença de lesão de aspecto tumoral, de $3 \mathrm{~cm}$, em topografia de cabeça de pâncreas. O quadro articular ainda não havia mostrado nenhuma melhora, e solicitou-se acompanhamento da gastroenterologia.

Posteriormente, realizou-se tentativa de biópsia de pâncreas via endoscópica, sem sucesso. O paciente evoluiu rapidamente, com queda significativa do estado geral, não havendo possibilidade de cirurgia, nem de quimioterapia, vindo a falecer em 2 meses.

\section{DISCUSSÃO}

Doenças pancreáticas (inflamatórias ou neoplásicas) têm sido associadas a uma entidade bem estabelecida, que inclui: necrose gordurosa subcutânea (paniculite) e $\operatorname{artropatia}^{(8)}$. O reconhecimento dessa entidade é relevante para o diagnóstico e terapêutica precoces, sobretudo em casos de doença pancreática oculta (como ocorreu nesse caso) ${ }^{(9)}$, visto que lesões de pele e manifestações articulares podem preceder sintomas abdominais da doença pancreática. Pancreatite, freqüentemente com pseudocistos, e carcinoma de células acinares são as principais doenças pancreáticas associadas, mas o espectro é ainda maior, incluindo cálculo e trauma pancreático, pancreatite isquêmica, fístula arteriovenosa do ducto pancreático, entre outras ${ }^{(8,10,11)}$.

O mecanismo envolvido na patogênese da necrose gordurosa subcutânea e periarticular ainda é pouco conhecido. Sugere-se que esteja relacionado à lipase pancreática oriunda da hipersecreção de células acinares tumorais, ou resultante de obstrução ductal em razão de pancreatite edematosa concomitante ${ }^{(8)}$. Uma vez na circulação sistêmica, ocorre autodigestão de gordura subcutânea periarticular ${ }^{(1,5)}$ e de outros sítios, onde, posteriormente, a lipase vem a ser ativada $^{(3,12,13)}$, desencadeando reação inflamatória.

A paniculite é uma lesão altamente específica para doença pancreática, particularmente carcinoma acinar de pâncreas, e denota pior prognóstico ${ }^{(9)}$. Clinicamente, manifesta-se principalmente como nódulos dolorosos, medindo cerca de 1 a $2 \mathrm{~cm}$, eritematosos, podendo surgir isolados ou difusos $^{(12)}$. Podem ocorrer em qualquer local do corpo, mas freqüentemente acometem membros inferiores ${ }^{(3,4,9)}$, sobretudo em área pré-tibial ${ }^{(6,11)}$ e parte inferior dos joelhos ${ }^{(14)}$. No caso relatado, observou-se essa preferência de localização dos nódulos. Observa-se, em outros relatos de casos, que a paniculite geralmente precede o diagnóstico de 1 a 7 meses, podendo ser um anúncio de doença metastática ${ }^{(9)}$, regredindo em 2 a 8 semanas, transformando-se em escaras pigmentadas. $\mathrm{Na}$ sua forma grave, podem-se tornar flutuantes ou ulcerativas ${ }^{(9,11)}$. O diagnóstico diferencial de tais nódulos inclui: doença de Weber-Christian, eritema nodoso, paniculite lúpica, vasculites e reação a drogas ${ }^{(4,6,7)}$. A histologia é diagnóstica, mostrando uma forma única de necrose gordurosa (paniculite primária) ${ }^{(8)}$. Os achados patológicos em lesões agudas incluem a presença de células anucleadas, com áreas de necrose gordurosa cercadas por células inflamatórias ${ }^{(1,9,13)}$. Tais características não estão presentes em outras causas de paniculite ${ }^{(6,12)}$.

Ainda em relação às manifestações clínicas, podem-se encontrar febre, eosinofilia, dor abdominal, náuseas e vômitos. $\mathrm{O}$ paciente em questão não apresentou sinais nem sintomas abdominais, o que, de certa forma, dificultou e retardou o diagnóstico da doença pancreática. Outros casos 
na literatura atentam para escassez de sintomas abdominais. História de etilismo crônico também foi fator comum à maioria dos relatos.

Embora não se tenha pesquisado no paciente relatado, $\mathrm{o}$ acometimento ósseo pode ocorrer, relacionando-se patologicamente à extensão da necrose gordurosa intramedular e à destruição trabecular óssea, cujo aspecto radiológico é citado como múltiplas lesões pequenas em região cortical de ossos longos, ocorrendo, geralmente, de modo indolor ${ }^{(1,6,10,14,15)}$.

A elevação sérica de enzimas pancreáticas verificada no relato e reportada em muitos casos reforça a patogênese da necrose gordurosa ${ }^{(1,8,11,12)}$. Estudos comparativos sugerem que tais enzimas causariam hidrólise no tecido adiposo periarticular e na medula óssea, liberando ácidos gordurosos, que, por sua vez, atuariam desencadeando o processo inflamatório, sobretudo nas manifestações extrapancreáticas $^{(8,10,11,12,16)}$.

A poliartrite é uma rara complicação de pancreatite ${ }^{(4)} \mathrm{e}$ carcinoma pancreático ${ }^{(17)}$. O envolvimento articular é variável, podendo ser mono ou poliarticular, manifestando-se concomitante ou separadamente dos nódulos subcutâneos,

\section{REFERÊNCIAS}

1. Virshup AM, Sliwinski AJ: Polyarthritis and subcutaneous nodules associated with carcinoma of the pancreas. Arthritis Rheum 16: 388-92, 1973.

2. Lucas PF, Owen TK: Subcutaneous fat necrosis, polyarthritis and pancreatic disease. Gut 3: 146-8, 1962.

3. Mullin GT, Caperton EM Jr, Crespin SR, Williams RC Jr: Arthritis and skin lesions resembling erythema nodosum in pancreatic disease. Ann Intern Med 68: 75-87, 1968.

4. Tannenbaum H, Anderson LG, Schur PH: Association of polyarthritis, subcutaneous nodules and pancreatic disease. J Rheumatol 2: 4-20, 1975.

5. Van Klaveren RJ, de Mulder PH, Boerbooms AM, et al.: Pancreatic carcinoma with polyarthritis, fat necrosis, and high serum lipase and trypsin activity. Gut 31(8): 953-5, 1990.

6. Watts RA, Kelly S, Hacking JC, Lonas D, Hazleman BL: Fat necrosis. An unusual cause of polyarthritis. J Rheumatol 20: 1432-5, 1993.

7. Sibrack LA, Gouterman IH: Cutaneous manifestations of pancreatic diseases. Cutis 21: 763-8, 1978.

8. Fine RM: Subcutaneous fat necrosis, pancreatitis and arthropathy. Intern J Dermatol 22: 575-6, 1983

9. Skarin A: Pancreatic panniculitis. J Clin Oncol 15: 3418-9, 1997. podendo preceder a dor abdominal ou qualquer outra evidência de doença pancreática ${ }^{(1)}$. $\mathrm{O}$ acometimento articular pode mimetizar a gota, febre reumática, sarcoidose, artrite infecciosa ou doenças do tecido conjuntivo ${ }^{(1,3)}$. A pesquisa diagnóstica deve, sempre que possível, incluir exame do líquido sinovial das articulações comprometidas, como também biópsia do nódulo. Sugere-se também a determinação de enzimas pancreáticas, ultra-sonografia de abdome total, principalmente em pacientes alcoólatras, com conhecida doença pancreática ${ }^{(1,18)}$.

A terapia que emprega antiinflamatórios não esteroidais, glicocorticóides e agentes imunossupressores se mostrou ineficaz para reverter o processo inflamatório, não interferindo na evolução dos nódulos subcutâneos, nem no processo articular ${ }^{(1,8,14)}$. No paciente relatado, realizou-se terapia com antiinflamatórios e glicocorticóides, após tratamento inicial com antibióticos, também sem melhora do processo inflamatório ou da dor.

Portanto, deve-se estar atento para a possibilidade diagnóstica de doença maligna pancreática, em vigência de paniculite e artrite, mesmo sem queixas abdominais.

10. Simkin PA, Brunzell JD, Wisner D, Fiechtner JJ, Carlin JS, Willkens RF: Free fatty acids in the pancreatitic arthritis syndrome. Arthritis Rheum 26: 127-32, 1983.

11. Palomo-Arellano A, Salvador-Fernandez M, Gil-Pascual B, Santome-Argibay F: Erythematous and tender subcutaneous nodules on lower extremities. Subcutaneous fat necrosis associated with pancreatic adenocarcinoma. Arch Dermatol 130(5): 649-52, 1994.

12. Wilson HA, Askari AD, Neuderhiser DH, et al.: Pancreatitis with artropathy and subcutaneous fat necrosis: evidence for the pathogenicity of lipolytic enzymes. Arth Rheum 26: 121-6, 1983.

13. Shalita AR, Wheatley V: Inhibition of pancreatic lipase by tetracyclines. J Invest Dermatol 54: 413-5, 1970.

14. Good AE, Schnitzer B, Kawanishi H, Demetropoulos KC, Rapp R: Acinar pancreatic tumor with metastatic fat necrosis. Digestive Diseases 21: 978-87, 1976.

15. Morita O, Ogose A, Hotta T, et al.: Pathological fractures due to intraosseous fat necrosis associed with pancreatitis. Rheumatology 42: 394-6, 2003.

16. Ferrari R, Wendelboe M, Ford PM, Corbett WEN, Anastassiades TP: Pancreatitis arthritis with periarticular fat necrosis. J Rheumatol 20: 1436-7, 1993.

17. Jackson SH, Savidge RS, Stein L, Varley H: Carcinoma of the pancreas associated with fat necrosis. Lancet 2: 962-7, 1952.

18. Francombe J, Kingsnorth AN, Tunn E: Panniculitis, arthritis and pancreatitis. J Rheumatol 34: 680-3, 1995. 